ICRR-Report-374-96-25

UT-758

hep-ph/9608405

\title{
Cosmological Axion Problem in Chaotic Inflationary Universe
}

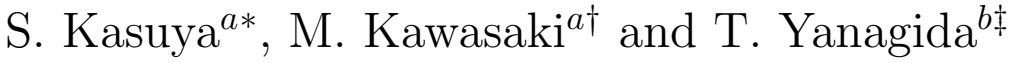 \\ ${ }^{a}$ Institute for Cosmic Ray Research, University of Tokyo, Tanashi, Tokyo 188, Japan \\ ${ }^{b}$ Department of Physics, School of Science, University of Tokyo, Tokyo 113, Japan
}

\begin{abstract}
We investigate two cosmological axion problems ( isocurvature fluctuations and domain-wall formation ) in chaotic inflationary universe. It is believed that these problems are solved if potential for the Peccei-Quinn scalar field is very flat. However, we find that too many domain walls are produced through parametric resonance decay of the Peccei-Quinn scalar field. Only the axion model with $N=1(N$ : QCD anomaly factor) is consistent with observations. We also point out that the flat potential is naturally obtained in a supersymmetric extension of the PecceiQuinn model. If Peccei-Quinn breaking scale $F_{a}$ is about $10^{12} \mathrm{GeV}$, this model predicts anisotropies of cosmic microwave background radiation due to the axion isocurvature fluctuations which may be detectable in future observations.
\end{abstract}

\footnotetext{
*kasuya@icrr.u-tokyo.ac.jp

$\dagger^{\dagger}$ kawasaki@icrr.u-tokyo.ac.jp

łyanagida@danjuro.phys.s.u-tokyo.ac.jp
} 


\section{Introduction}

The axion [1, 2, 3, [4] is a Nambu-Goldstone boson associated with breaking of the PecceiQuinn symmetry which was invented as a solution to the strong CP problem in QCD [5]. The Peccei-Quinn symmetry breaking scale $F_{a}$ is stringently constrained by laboratory experiments, astrophysics and cosmology: allowed range of $F_{a}$ lies between $10^{10} \mathrm{GeV}$ and $10^{12} \mathrm{GeV}[6]$. The axion is also cosmologically attractive since it can be a cold dark matter if $F_{a}$ takes the higher values $\sim 10^{12} \mathrm{GeV}$.

Inflationary universe [7, 8] was proposed to solve various problems in the standard cosmology (e.g. flatness and horizon problems). There have been constructed many inflation models since then. The chaotic inflation model [9] seems the most attractive candidate among them that realizes naturally the inflationary universe. However, when we consider the axion in the chaotic inflationary universe, we are confronted with two serious cosmological problems, both of which are associated with large quantum fluctuations generated in the exponentially expanding phase (i.e., inflationary epoch) of the early universe. One is the domain wall problem [10]. In the inflationary universe fluctuations of the axion field $a(x)$ are given by $\delta a=H /(2 \pi)$ where $H$ is the Hubble constant. Since a phase $\theta_{a}$ of the Peccei-Quinn scalar field is related to the axion field $a(x)$ by $\theta_{a}=a / F_{a}$, the fluctuations of $\theta_{a}$ is given by

$$
\delta \theta_{a}=\frac{H}{2 \pi F_{a}} .
$$

In the chaotic inflation model, $H \simeq 10^{14} \mathrm{GeV}$ [11] is required to explain the anisotropies of cosmic microwave background radiation (CBR) observed by COBE [12]. Then, the fluctuations of the phase $\theta_{a}$ become $O(1)$ for $F_{a} \lesssim 10^{12} \mathrm{GeV}$, which means that the phase is quite random during the inflation. Therefore, when the universe cools down to about $1 \mathrm{GeV}$ and the axion potential is formed, the axion sits at different positions of the potential in different regions of the universe. Since the axion potential has $N$ discrete minima ( $N$ : QCD anomaly factor), domain walls are produced [13]. The domain wall with 
$N=1$ is a disk-like object whose boundary is an axionic string and it collapses quickly due to its surface tension. Thus the domain wall with $N=1$ is cosmologically harmless. However, the domain wall with $N \geq 2$ is disastrous because it forms a complicated network with an axionic string and dominates the energy density of the universe quickly [14].

The second problem is that the quantum fluctuations for the axion field cause too large anisotropies of CBR [15, 16, 17, 18, 19]. Since the axion does not have a potential during the inflation, the axion fluctuations $\delta a$ do not contribute to the energy density of the universe. In this sense, the axion fluctuations are isocurvature. After the axion acquires a mass $m_{a}$, its fluctuations become density fluctuations given by $\delta \rho_{a} / \rho_{a} \sim \delta \theta_{a} / \theta_{a}$ which causes the CBR temperature fluctuations $\delta T / T \sim \delta \theta_{a} / \theta_{a}$. From eq.(11), we see that the produced CBR anisotropies are $O(1)$ which contradicts the observation.

It has been pointed out in ref.[17, 18] that these problems are simultaneously solved if the potential for the Peccei-Quinn scalar field is very flat. In this case it is natural that the Peccei-Quinn scalar field $\Phi$ takes a very large value $\sim M_{\mathrm{pl}}$ during the inflation. Here, $M_{\mathrm{pl}}$ is the Planck mass $\left(M_{\mathrm{pl}}=1.2 \times 10^{19} \mathrm{GeV}\right)$. The axion field $a(x)$ is defined as

$$
\begin{aligned}
\Phi(x) \equiv & \phi(x) \exp (i a(x) /|\langle\phi\rangle|), \\
& \phi(x): \text { real }, \quad-\infty<\phi<\infty, \\
& a(x): \text { real }, \quad-\pi / 2 \leq a(x) /|\langle\phi\rangle| \leq \pi / 2 .
\end{aligned}
$$

In the true vacuum $\phi$ takes $|\langle\phi\rangle|=F_{a}$. But the $\phi$ takes most likely $|\langle\phi\rangle| \sim M_{\mathrm{pl}}$ at the inflationary epoch. From eq.(1) it is clear that the phase fluctuations $\delta \theta_{a}$ are suppressed as $\delta \theta_{a} \simeq H /\left(2 \pi M_{\mathrm{pl}}\right)$. Therefore the isocurvature fluctuations are suppressed by a factor $F_{a} / M_{\mathrm{pl}}$. In ref. [19], cosmological effects of the axionic isocurvature density fluctuations have been investigated in details by calculating spectra for matter fluctuations and $\delta T / T$, and it has been found that the effects of the isocurvature fluctuations are consistent with observations in a large parameter region.

As for the domain wall problem, it is naively expected that the same mechanism 
that suppresses the isocurvature fluctuations does also suppress the production of domain walls: i.e. the large effective " $F_{a} " \equiv|\langle\phi\rangle|_{\text {inflation }} \sim M_{\mathrm{pl}}$ during the inflation almost fixes the phase of $\Phi$ which reduces the domain-wall production rate to negligible amount. In fact, the $\Phi$ begins to oscillate after the inflation with its phase being fixed. However, the homogeneous Peccei-Quinn field decays into axions as well as $\phi$ particles through parametric resonances. The emitted axions induce large fluctuations of the axion field (i.e. the phase $\delta \theta_{a}$ ) and produce a large number of domain walls.

In this letter we study the above cosmological axion problems ( isocurvature-fluctuation and domain-wall problems ) in chaotic inflationary universe. We show that the domain walls are still produced through the parametric resonance decay even if we take a very flat potential for the Peccei-Quinn scalar. The domain wall problem is only avoided for the model with $N=1$. Furthermore, we also show that the flat potential for $\Phi$ required to solve the isocurvature-fluctuation problem is naturally obtained in a supersymmetric (SUSY) extension of the Peccei-Quinn model. Namely, the presence of flat directions is a generic feature in SUSY theories and the masses of the fields corresponding to the flat directions only comes from soft SUSY breaking terms which are of order the weak scale. Therefore the flat potential for $\Phi$ is naturally realized in the framework of SUSY.

\section{Domain Wall Problem for a Quartic Potential}

Let us first consider the case for a scalar potential given by

$$
V(\Phi)=\frac{g}{4}\left(|\Phi|^{2}-F_{a}^{2}\right)^{2}=\frac{g}{4}\left(\phi^{2}-F_{a}^{2}\right)^{2}
$$

with $g \ll 1$. In the inflationary epoch, the $\phi$ slowly evolves as

$$
\phi \simeq\left(\frac{\lambda}{g}\right)^{1 / 2} \chi,
$$

where we have assumed that the potential for the inflaton $\chi$ is $\lambda \chi^{4} / 4$. Isocurvature

fluctuations for cosmologically relevant scales are generated when $\chi \simeq 4 M_{\mathrm{pl}}$. Therefore 
the CBR anisotropies from the axion isocurvature fluctuations is given by 1

$$
\frac{\delta T}{T} \sim \frac{\delta \rho_{a}}{\rho_{a}} \sim \sqrt{\frac{2}{3 \pi}} g^{1 / 2}\left(\frac{\chi}{M_{\mathrm{pl}} \theta_{a}}\right) \simeq 2 \frac{g^{1 / 2}}{\theta_{a}} .
$$

Using a COBE constraint $\delta T / T \lesssim 10^{-5}$, we get $g \lesssim 10^{-11}$ for $\theta_{a} \sim O(1)$. I Notice that the coupling constant $\lambda$ for the inflaton is already very small as $\lambda \sim 10^{-13}$ [11].

Next we consider domain wall production with the potential (3). The Peccei-Quinn field $\Phi$ starts to oscillate after the inflation. We only consider the evolution of $\Phi$ in the "radial" direction $\phi(x)$ with its phase being fixed since the quantum fluctuations of the axion phase $\delta \theta_{a} \equiv \delta a /\langle\phi\rangle$ are negligiblly small when $|\langle\phi\rangle| \sim M_{\mathrm{pl}}$ as discussed in the introduction. As easily seen from eq.(3) the "radial" potential has two minima $\left(\phi= \pm F_{a}\right)$. P Since the initial potential energy density of $\phi$ is large, the $\phi$ oscillates beyond the potential hill at $\phi=0$. The $\phi$ loses its energy through cosmic expansion and particle production and settles down to one of the potential minima eventually.

If the dynamics after inflation is purely classical, the final value of $\phi\left(F_{a}\right.$ or $\left.-F_{a}\right)$ depends on the initial value of $\phi$. However, it has been recently pointed out that the oscillating coherent field quickly losses its energy through violent particle production due to parametric resonance [20]. The parametric resonance decay occurs during the $\phi$ oscillation. In the present model, only a self-production (e.g. $\langle\phi\rangle+\langle\phi\rangle \rightarrow \Phi+\Phi)$ is effective. (Notice that the "radial" oscillation induces excitations of the phase (=axions) as well as $\phi$.$) In this case the classical picture does not apply at all. The important$ fact is that the quantum fluctuations due to the emitted $\phi$ and axion particles becomes

\footnotetext{
${ }^{1}$ For a more accurate analysis including comparison with the most recent observations, see ref. 19]

${ }^{2}$ Even if one assumes the inflaton potential $\sim m_{\chi}^{2} \chi^{2} / 2$, one gets essentially the same conclusion as in the text.

${ }^{3}$ Note that in the conventional notation of the axion field $\Phi=|\langle\Phi\rangle| e^{i a(x) /|\langle\Phi\rangle|}$ the two minima correspond to $\theta_{a}^{0}$ and $\theta_{a}^{0}+\pi$ where $\theta_{a}^{0}$ is the initial value of the axion phase.

${ }^{4}$ In principle, the $\Phi$ can couple to other fields $\eta$. Although the mass of $\eta$ is large $\sim O\left(F_{a}\right)$ in the true vacuum of $\Phi$, the coherent $\Phi$ field also decays into $\eta$ particles through the parametric resonance. However, this does not change the conclusion in this paper as will be noted in the end of this section.
} 
quite large. 向 Contributions of the quantum fluctuations $\delta \phi$ and $\delta a=|\phi| \delta \theta_{a}$ to the energy density of $\Phi$ are $\sim g(\delta \phi)^{2} M_{\mathrm{pl}}^{2}$ [20] and $\sim g\left(\delta \theta_{a}\right)^{2} M_{\mathrm{pl}}^{4}$, f respectively. Since most of the initial oscillation energy $g M_{\mathrm{pl}}^{4}$ is transferred to $\Phi$ particles through the parametric resonance, we expect that the fluctuations of $\phi$ and $\theta_{a}$ are given by $\delta \phi \sim M_{\mathrm{pl}}$ and $\delta \theta_{a} \sim 1$, respectively, which leads to production of domain walls.

For more quantitative analysis, we need to solve equations for modes of the fluctuations $\delta \phi_{k}$ and $\delta a_{k}$ which correspond to $\phi$ particle and axion with momentum $k$, respectively. However it is more convenient to use two field $X$ and $Y$ which are defined by $X \equiv \operatorname{Re}(\Phi)$ and $Y \equiv \operatorname{Im}(\Phi)$ with initial condition $X(0)=|\phi(0)|$ and $Y(0)=0$. Then the fluctuations of $\phi$ and $\theta_{a}$ are approximately given by $\delta \phi \simeq \delta X$ and $\delta \theta_{a} \simeq \delta Y /|X|$. The evolutions of $X, \delta X$ and $\delta Y$ are described by

$$
\begin{aligned}
\ddot{X}+g X\left(X^{2}-F_{a}^{2}\right) & =-3 g\left\langle(\delta X)^{2}\right\rangle X-g\left\langle(\delta Y)^{2}\right\rangle X, \\
\delta \ddot{X}_{k}+\left[k^{2}-g F_{a}^{2}+3 g X^{2}\right] \delta X_{k} & =-3 g\left\langle(\delta X)^{2}\right\rangle \delta X_{k}-g\left\langle(\delta Y)^{2}\right\rangle \delta X_{k}, \\
\delta \ddot{Y}_{k}+\left[k^{2}-g F_{a}^{2}+g X^{2}\right] \delta Y_{k} & =-g\left\langle(\delta X)^{2}\right\rangle \delta Y_{k}-3 g\left\langle(\delta Y)^{2}\right\rangle \delta Y_{k},
\end{aligned}
$$

where we have used the mean field approximation $\left((\delta X)^{3} \simeq 3 \delta X\left\langle(\delta X)^{2}\right\rangle, \cdots\right)$ and neglected the cosmological expansion.] Before we present results of the full numerical integration of the above equations, we briefly discuss instability of the equations for $\delta X$ and $\delta Y$. When the amplitude of the $X$ oscillation is much larger than $F_{a}$, a solution to eq.(6) is approximately given by

$$
X \simeq Z \sin (c \sqrt{g} Z t)
$$

\footnotetext{
${ }^{5}$ Notice that the wavelengths of the fluctuations induced by the decay are much shorter than the horizon scale. Therefore, these fluctuations do not make any contribution to the fluctuations with wavelengths relevant to large scale structures of the universe.

${ }^{6}$ This energy density comes from the kinetic term of the axion field.

${ }^{7}$ The cosmological expansion reduces the effect of parametric resonance in general. However we find that the reduction effect is not large enough to avoid the conclusion in this paper.
} 
where $c=\mathcal{O}(1)$ and $Z$ is the amplitude of the oscillation. Using this solution and neglecting back reactions, eqs.(7) and (8) become well-known Mathieu equation:

$$
\begin{aligned}
\delta X_{k}^{\prime \prime}+\left[A_{X}(k)-2 q_{X} \cos (2 z)\right] \delta X_{k} & \simeq 0 \\
\delta Y_{k}^{\prime \prime}+\left[A_{Y}(k)-2 q_{Y} \cos (2 z)\right] \delta Y_{k} & \simeq 0
\end{aligned}
$$

where $A_{X}=\frac{k^{2}-g F_{a}^{2}}{c^{2} g Z^{2}}+2 q_{X}, q_{X}=\frac{3}{4 c^{2}}, A_{Y}=\frac{k^{2}-g F_{a}^{2}}{c^{2} g Z^{2}}+2 q_{Y}, q_{Y}=\frac{1}{4 c^{2}}, z=c \sqrt{g} Z t$ and a prime denotes a derivative with respect to $z$. For $q_{X, Y} \lesssim 1$, the Mathieu equation has instability for $k$ which satisfies $A(k) \sim 1,4,9, \cdots$. Since $A_{X}(k)>1$ the strongest instability (resonance) for $\delta X_{k}$ occurs in the second instability band, i.e. $A_{X}(k) \sim 4$. On the other hand the resonance for $\delta Y_{k}$ occurs in the first instability band and the instability is stronger than that for $\delta X_{k}$. Since $\delta Y_{k}$ roughly corresponds to the axion with momentum $k$ it is expected that the homogeneous $X$ field decays dominantly into axions. However, as the fluctuations increase, back reactions, i.e., terms in RHS of eqs.(7) and (8), become significant and the instability is weakened. To see the evolution of the fluctuations with back reactions, we need numerical calculations as shown below.

Precise time evolution of the fluctuations is obtained by full numerical integration of eqs.(6) - (8) and results are shown in Fig. 1 - 1 . As seen in Fig. 2 and (3, the fluctuations of both $X$ and $Y$ increase through the parametric resonances. Since the instability of $\delta Y$ is stronger, the fluctuations of $Y$ increase faster than those of $X$. As the fluctuations increase, the back reactions become important and weaken the instability and finally the fluctuations become almost constant. As is expected, the final fluctuations are large and, in particular, the phase fluctuations are of order $1\left(\delta \theta_{a} \sim 1\right)$, which results in the production of too many domain walls (see Fig. 目).

The above mechanism for producing domain walls seems quite general. In fact we have solved equations for the fluctuations with a potential $V=g / M_{\mathrm{pl}}^{2}\left(|\Phi|^{2}-F_{a}^{2}\right)^{3}$ and obtain almost the same result. We also consider interaction between $\Phi$ and other scalar 
fields $\eta: \mathcal{L}_{\text {int }}=-f|\Phi|^{2}|\eta|^{2}$. If the coupling $f$ is larger than $g$, the $\Phi$ field first decays into $\eta$-particles. However, the back reactions of $\eta$-particles suppress the resonance decay when $\delta \eta \sim M_{p l}[20$. Then the remaining $\Phi$ field may decay into $\phi$-particles and axions through the parametric resonance, which results in large phase fluctuations $(\sim O(1))$. Thus the production of $\eta$ particles cannot suppress the violent fluctuations of the axion phase. Therefore, the production of domain walls may not be avoided in the chaotic inflationary universe. Since the produced domain walls dominate the density of the universe for the axion model with $N \geq 2$, only the model with $N=1$ is allowed. Fortunately, the $N=1$ model is easily constructed in a hadronic axion model for example [21].

\section{$3 \quad$ Supersymmetric Potential}

We now consider a SUSY extension of a hadronic axion model. We take the following simple superpotential:

$$
W=h\left(\Psi_{+} \Psi_{-}-F_{a}^{2}\right) \Psi_{0}
$$

where $\Psi_{+}, \Psi_{-}$and $\Psi_{0}$ are chiral superfields with Peccei-Quinn charges $+1,-1$ and 0 , respectively, and the coupling constant $h$ is assumed to be $\sim O(1) .9$ Then the scalar potential is written as

$$
V_{\mathrm{SUSY}}=h^{2}\left|\Phi_{+} \Phi_{-}-F_{a}^{2}\right|^{2}+h^{2}\left(\left|\Phi_{+}\right|^{2}+\left|\Phi_{-}\right|^{2}\right)\left|\Phi_{0}\right|^{2}
$$

where $\Phi_{+}, \Phi_{-}$and $\Phi_{0}$ are scalar components of superfields $\Psi_{+}, \Psi_{-}$and $\Psi_{0}$, respectively. Here we should note that there are flat directions that satisfy

$$
\Phi_{+} \Phi_{-}=F_{a}^{2}, \quad \Phi_{0}=0 .
$$

\footnotetext{
${ }^{8}$ Possible trilinear coupling contributing to $\Phi$ decay is $\mathcal{L}_{\text {int }}=-f^{\prime} \Phi \eta^{2}$. This interaction, however, gives unbounded potential at the vacuum $|\Phi|=F_{a}$ and hence we discard this possibility.

${ }^{9}$ We also assume a pair of quark $Q$ and antiquark $\bar{Q}$ whose Peccei-Quinn charges are $-1 / 2$. This charges are chosen so that $Q$ and $\bar{Q}$ have a Yukawa coupling $W=f Q \bar{Q} \Psi_{+}$. This model has the QCD anomaly factor $N=1$.
} 
We also add soft SUSY breaking terms to the potential. Then, the scalar potential $V$ that we should study is

$$
V=V_{\mathrm{SUSY}}+m_{+}^{2}\left|\Phi_{+}\right|^{2}+m_{-}^{2}\left|\Phi_{-}\right|^{2}+m_{0}^{2}\left|\Phi_{0}\right|^{2}
$$

where $m_{ \pm, 0}$ are soft masses of $O(100 \mathrm{GeV})$.

We discuss cosmological evolution of these scalar fields. The chaotic situation at the Planck time may lead to large initial values of order $M_{\mathrm{pl}}$ for $\Phi_{+}, \Phi_{-}$and $\Phi_{0}$. The soft masses can be negligible at the inflation epoch since they are much smaller than the expansion rate of the universe $\left(H \sim 10^{14} \mathrm{GeV}\right)$. $⿴ 囗 十$ Therefore these fields roll down into the flat direction given by eq.(14). However, one scalar field, either $\Phi_{+}$or $\Phi_{-}$, may stay at the initial position, i.e., $\left|\Phi_{+}\right|$or $\left|\Phi_{-}\right| \sim M_{\mathrm{pl}}$. We assume that it is the case and take, for definiteness, $\left|\Phi_{+}\right| \sim M_{\mathrm{pl}}$ and eliminate $\Phi_{-}$and $\Phi_{0}$ using eq.(14). Then, the effective lagrangian for $\Phi_{+}$is written as

$$
\begin{aligned}
L & =L_{\text {kin }}-V, \\
L_{\text {kin }} & =\frac{\left|\Phi_{+}\right|^{4}+F_{a}^{4}}{\left|\Phi_{+}\right|^{4}} \partial_{\mu} \Phi_{+}^{*} \partial^{\mu} \Phi_{+}, \\
V & =m_{+}^{2}\left|\Phi_{+}\right|^{2}+m_{-}^{2} F_{a}^{4}\left|\Phi_{+}\right|^{-2} .
\end{aligned}
$$

Since the potential (18) is very steep at $\Phi_{+}=0$ and the kinetic term is always positive, the energy conservation never allows the $\Phi_{+}$to pass through the origin $\left(\Phi_{+}=0\right)$. Thus, this potential has effectively only one minimum. From the above lagrangian we obtain equations of motion for the homogeneous field $\Phi_{+}$and phase fluctuations $\delta \theta$ :

$$
\begin{aligned}
\varphi^{\prime \prime}-2 \frac{\varphi^{\prime 2}}{\left(\varphi^{4}+1\right) \varphi}+\frac{\varphi^{4}-\left(m_{+} / m_{-}\right)^{2}}{\varphi^{4}+1} \varphi & =0, \\
\theta_{k}^{\prime \prime}+k^{2} \theta_{k}+\frac{2(\varphi-1)}{\varphi(\varphi+1)} \varphi^{\prime} \theta_{k}^{\prime} & =0,
\end{aligned}
$$

\footnotetext{
${ }^{10}$ At the inflation epoch, non-vanishing vacuum energy density breaks SUSY and may generally give scalar masses of the order of the Hubble constant $H$ [22]. However, there exist a class of supergravity models where such scalar masses are negligible compared with $H$ [23]. We assume such a class of models in this paper.
} 
where the prime denotes $d /\left(m_{+} d t\right), \varphi \equiv\left|\Phi_{+}\right| / F_{a}$ and $\theta_{k} \sim \int d x^{3} \delta \theta e^{i k x}$. In eq.(19) the back reactions of $\delta \theta$ are assumed to be neglected. Although eq.(20) looks different from the Mathieu equation, numerical calculations show that it has indeed strong instability (see Fig. 50). Therefore we expect that the parametric resonance induces large phase fluctuations and results in the production of domain walls. However these domain walls are cosmologically harmless since we consider the hadronic axion model with $N=1$ as discussed in the previous section.

Let us now estimate isocurvature fluctuations of the axion in the SUSY model. First we rewrite $\Phi_{+}$and $\Phi_{-}$as

$$
\Phi_{ \pm}=v_{ \pm} \exp i \frac{a_{ \pm}}{\sqrt{2}\left|v_{ \pm}\right|}, \quad-\infty<v_{ \pm}<\infty .
$$

We also define the fields $a$ and $b$ by

$$
\begin{aligned}
a & =\frac{v_{+}}{\left(v_{+}^{2}+v_{-}^{2}\right)^{1 / 2}} a_{+}-\frac{v_{-}}{\left(v_{+}^{2}+v_{-}^{2}\right)^{2}} a_{-}, \\
b & =\frac{v_{-}}{\left(v_{+}^{2}+v_{-}^{2}\right)^{1 / 2}} a_{+}+\frac{v_{+}}{\left(v_{+}^{2}+v_{-}^{2}\right)^{1 / 2}} a_{-} .
\end{aligned}
$$

From eq.(13), the potential $V_{b}$ for $b$ is given by

$$
V_{b}=-2 h^{2} F_{a}^{2} v_{+} v_{-} \cos \left(\frac{\left(v_{+}^{2}+v_{-}^{2}\right)^{1 / 2}}{\sqrt{2} v_{+} v_{-}} b\right) \text {. }
$$

Thus the mass of $b$ is $\sim\left(v_{+}^{2}+v_{-}^{2}\right)^{1 / 2} \sim M_{\mathrm{pl}} \sim 10^{19} \mathrm{GeV}$, which is much larger than the Hubble constant during the inflation and hence $b$ quickly becomes zero at the inflation epoch. On the other hand, the potential for $a$ is flat and is regarded as the axion. At the inflation epoch, the quantum fluctuations of $a$ are given by, assuming $v_{+} \gg v_{-}$,

$$
\delta a \simeq \delta a_{+} \simeq \frac{H}{2 \pi}
$$

Since the mass of $b$ is very large compared with $H$, the quantum fluctuations of $b$ are negligible and hence $\delta a_{-} \simeq-\left(v_{-} / v_{+}\right) \delta a_{+}$. Therefore, the fluctuations for phases $\left(\theta_{ \pm}\right)$of $\Phi_{ \pm}$are given by

$$
\delta \theta_{ \pm} \equiv \frac{\delta a_{ \pm}}{\sqrt{2} v_{ \pm}} \simeq \pm \frac{H}{2 \sqrt{2} \pi v_{+}}
$$


Since $v_{+} \sim M_{\mathrm{pl}}$ and $H \simeq 10^{14} \mathrm{GeV}$, fluctuations of the phase $\theta_{ \pm}$are quite small ( $\delta \theta_{ \pm} \sim$ $\left.10^{-6}-10^{-5}\right)$. After the axion obtains a mass, the phase fluctuations result in isocurvature density fluctuations and produce the CBR anisotropies as explained in the non-SUSY case. When $\Phi_{+}$and $\Phi_{-}$settle down to the true minimum of the potential (i.e., $v_{+} \sim v_{-} \sim F_{a}$ ), the axion field is written as $a \sim a_{+}-a_{-}$and its fluctuation $\delta a$ is given by $\delta a \sim F_{a} \delta \theta_{+}$, which gives $\delta \rho_{a} / \rho_{a} \sim \delta \theta_{+} / \theta_{+}$. Thus the $\delta T / T$ is predicted as $10^{-6}-10^{-5}$, which is consistent with the COBE observation. In particular, if $F_{a} \sim 10^{12} \mathrm{GeV}$, the predicted CBR anisotropies due to the isocurvature density fluctuations are large enough to be detected by future observations as stressed in ref. [19].

In SUSY Peccei-Quinn models, there exists an axino which is a supersymmetric partner of the axion. The mass of the axino is presumed to be the SUSY scale, i.e. $100 \mathrm{GeV}$ $-1 \mathrm{TeV}$. The axino of such a large mass is cosmologically harmless since it decays into lighter particles quickly.

\section{Conclusion}

In summary, we have investigated two cosmological axion problems (isocurvature fluctuations and domain-wall formation) in chaotic inflationary universe. The isocurvaturefluctuation problem is solved if potential for the Peccei-Quinn scalar field is very flat. However, we have found that too many domain walls are produced through the parametric resonance decay of the Peccei-Quinn scalar field and only the model with $N=1$ is allowed. We have also pointed out that the flat potential which is necessary to solve the isocurvature-fluctuation problem is naturally obtained in a SUSY extension of the PecceiQuinn model. Namely, we do not need any small coupling constant in the SUSY model for obtaining such a flat potential. On the other hand a very small coupling constant $\left(g \lesssim 10^{-11}\right)$ is necessary in non SUSY Peccei-Quinn models. 


\section{References}

[1] R.D. Peccei and H.R. Quinn, Phys. Rev. Lett. 38 (1977) 1440.

[2] S. Weinberg, Phys. Rev. Lett. 40 (1978) 223;

F. Wilczeck, Phys. Rev. Lett. 40 (1978) 279.

[3] J.E. Kim, Phys. Rev. Lett. 43 (1979) 103;

M. Shifman, A. Vainshtein and V. Zakharov, Nucl. Phys. B166 (1980) 493.

[4] M. Dine, W. Fischler and M. Srednicki, Phys. Lett. B104 (1981) 199.

[5] G. 't Hooft, Phys. Rev. Lett. 37 (1976) 8.

[6] E.W. Kolb and M.S. Turner, The Early Universe, Addison-Wesley, (1990).

[7] A.H. Guth, Phys. Rev. D23 (1981) 347.

[8] K. Sato, Mon. Not. R. astr. Soc. 195 (1981) 467.

[9] A.D. Linde, Phys. Lett. B129 (1983) 177.

[10] D.H. Lyth, Phys. Rev. D45 (1992) 3394;

D.H. Lyth and E.D. Stewart, Phys. Rev. D46 (1992) 532.

[11] D.S. Salopek, Phys. Rev. Lett. 69 (1992) 3602.

[12] G.F. Smoot et al., Astrophys. J. 396 (1992) L1.

[13] P. Sikivie, Phys. Rev. Lett. 48 (1982) 1156.

[14] B.S. Ryden, W.H. Press and D.N. Spergel, Astrophys. J. 375 (1990) 293.

[15] M.S. Turner and F. Wilczek, Phys. Rev. Lett. 66 (1991) 5;

M. Axenides, R. Brandenberger and M.S. Turner, Phys. Lett. B126 (1983) 178; 
P.J. Steinhardt and M.S. Turner, Phys. Lett. B129 (1983) 51;

D. Seckel and M.S. Turner, Phys. Rev. D32 (1985) 3178;

L.A. Kofman and A.D. Linde, Nucl. Phys. B282 (1987) 55.

[16] D.H. Lyth, Phys. Lett. 236 (1990) 408.

[17] A.D. Linde and D.H. Lyth, Phys. Lett. B246 (1990) 353.

[18] A.D. Linde, Phys. Lett. B259 (1991) 38.

[19] M. Kawasaki, N. Sugiyama and T. Yanagida, hep-ph/9512368, Phys. Rev. D (1996) in press.

[20] L. Kofman, A.D. Linde and A. Strarobinsky, Phys. Rev. Lett. 73 (1994) 3195;

L. Kofman, A.D. Linde and A. Strarobinsky, Phys. Rev. Lett. 76 (1996) 1011;

D. Boyanovsky, H.J.de Vega, R. Holman, D.-S. Lee and A. Singh, Phys. Rev. D51 (1995) 4419;

M. Yoshimura, Prog. Theor. Phys. 94 (1995) 873;

Y. Shtanov, J. Traschen and R. Brandenberger, Phys. Rev. D51 (1995) 5438;

S.Yu. Khlebnikov and I.I. Tkachev, Phys. Rev. Lett. 77 (1996) 219.

[21] J.E. Kim, Phys. Rep. C150 (1987) 1.

[22] M. Dine, L. Randall and S. Thomas, Phys. Rev. Lett. 75 (1995) 398.

[23] M. Gaillard, H. Murayama and K.A. Olive, Phys. Lett. B335 (1995) 71. 


\section{Figure Captions}

Fig.1 Time evolution of the fluctuations of $X . z$ is the time in units of $(c \sqrt{g} Z(0))^{-1}$ where $c$ is the $O(1)$ constant, $g$ is the self-coupling constant and $Z(0)$ is the initial amplitude of the oscillation of the Peccei-Quinn scalar field. We take $X(0)=M_{\mathrm{pl}} / 3$, and $g=10^{-13}$.

Fig.2 Time evolution of the fluctuations of $\delta X$.

Fig.3 Time evolution of the fluctuations of $\delta Y$.

Fig.4 Time evolution of the fluctuations of $\theta_{a}$. We define $\delta \theta \operatorname{as} \arctan \left(\sqrt{(\delta Y)^{2}} /|X|\right)$.

Fig.5 Time evolution of the phase fluctuations with $k=0.5 m_{+}$. We take $\left|\Phi_{+}(0)\right|=$ $10 F_{a}$ and $m=m_{+}=m_{-}$. This figure shows clearly the instability of the phase fluctuations. 


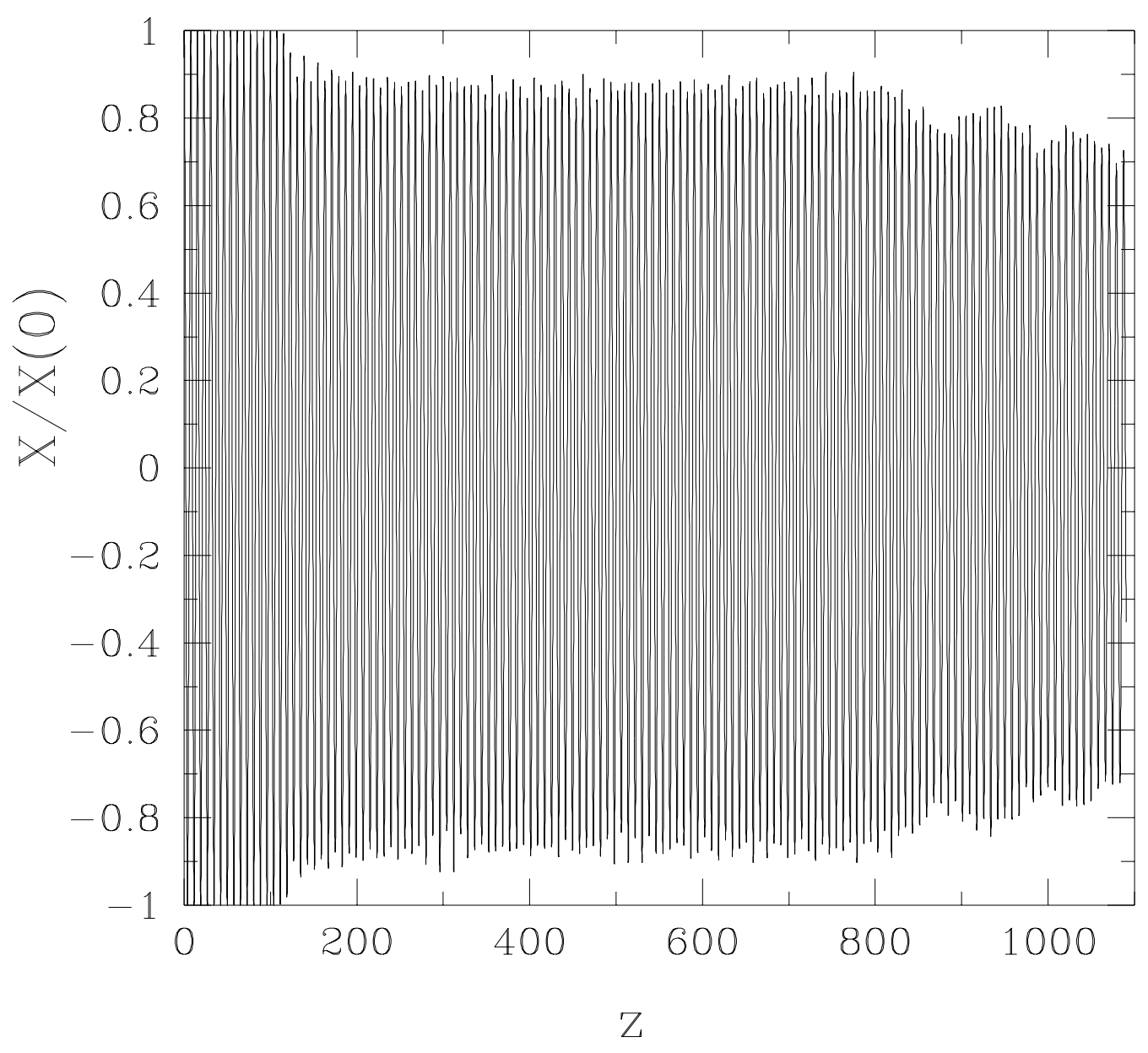

Figure 1: Time evolution of the fluctuations of $X . z$ is the time in units of $(c \sqrt{g} Z(0))^{-1}$ where $c$ is the $O(1)$ constant, $g$ is the self-coupling constant and $Z(0)$ is the initial amplitude of the oscillation of the Peccei-Quinn scalar field. We take $X(0)=M_{\mathrm{pl}} / 3$, and $g=10^{-13}$. 


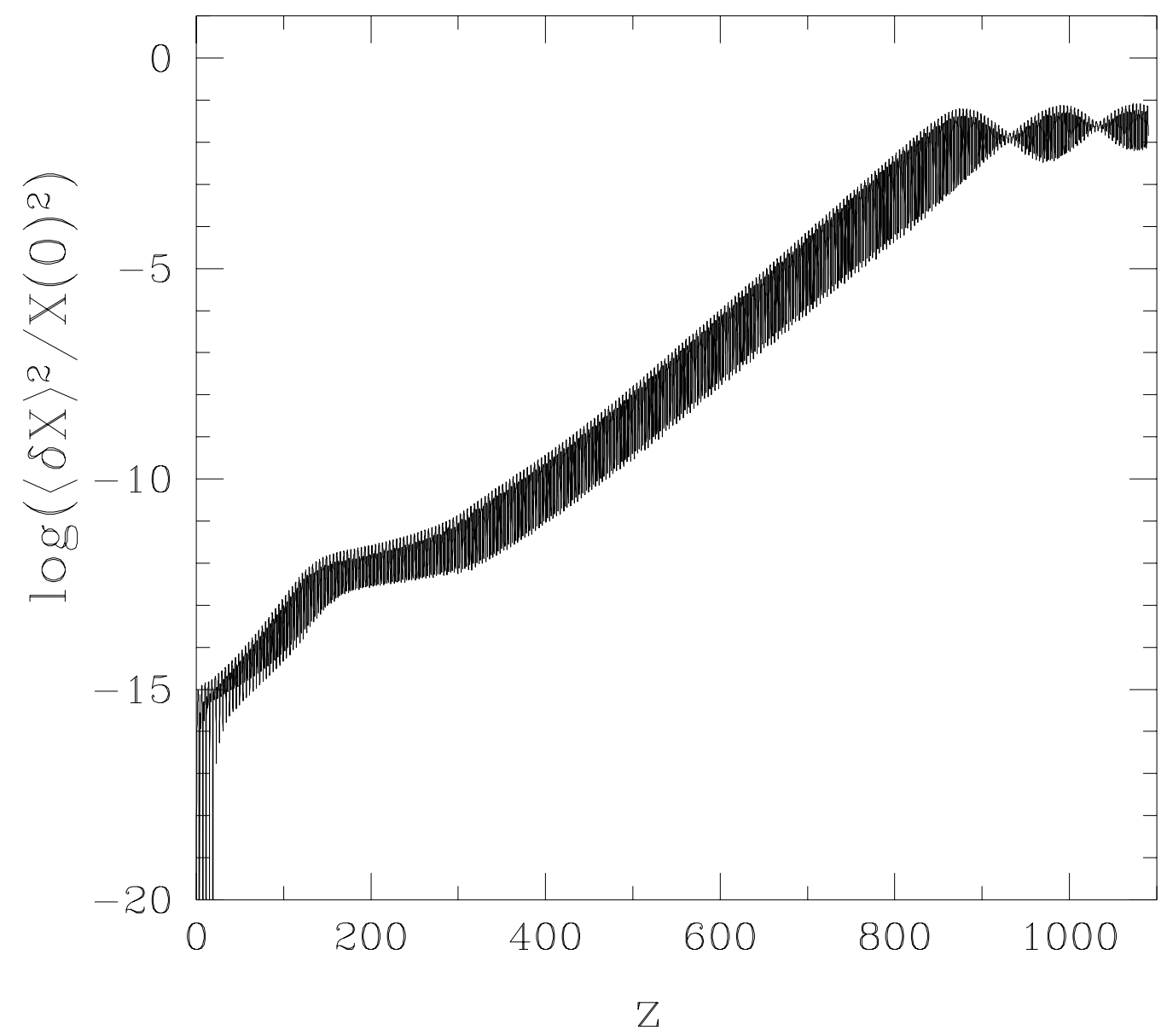

Figure 2: Time evolution of the fluctuations of $\delta X$. 


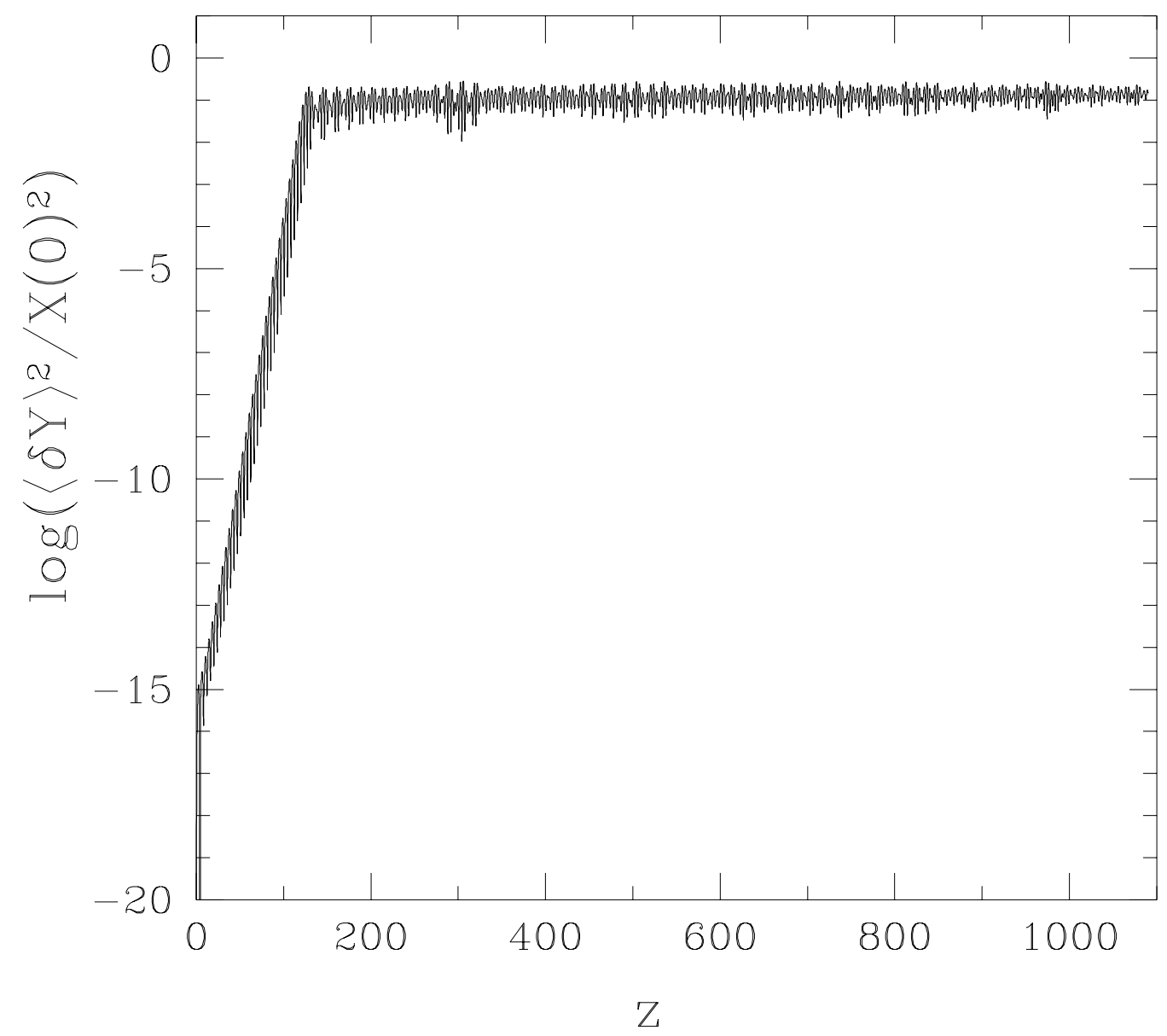

Figure 3: Time evolution of the fluctuations of $\delta Y$. 


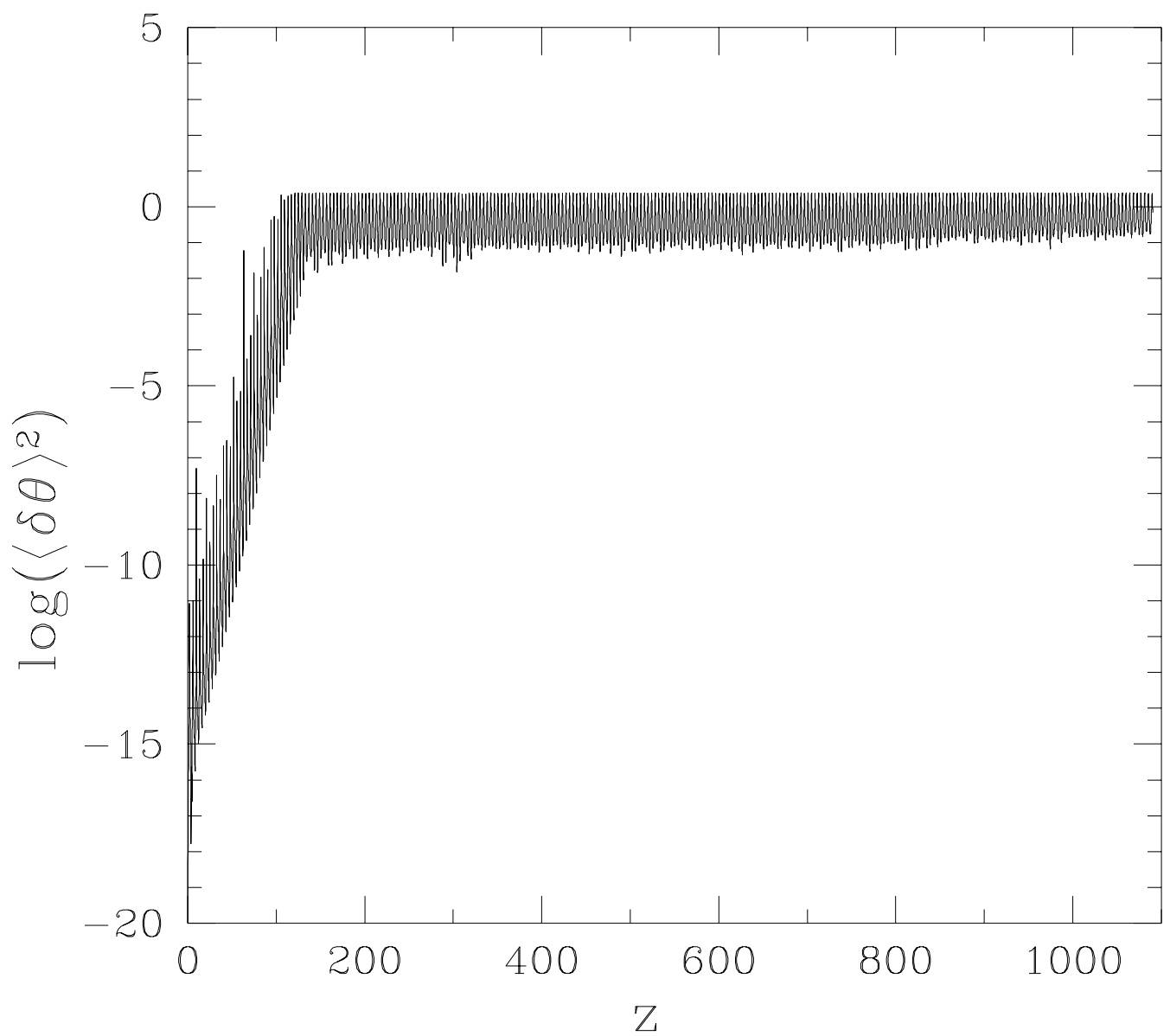

Figure 4: Time evolution of the fluctuations of $\theta_{a}$. We define $\delta \theta \operatorname{as} \arctan \left(\sqrt{(\delta Y)^{2}} /|X|\right)$. 


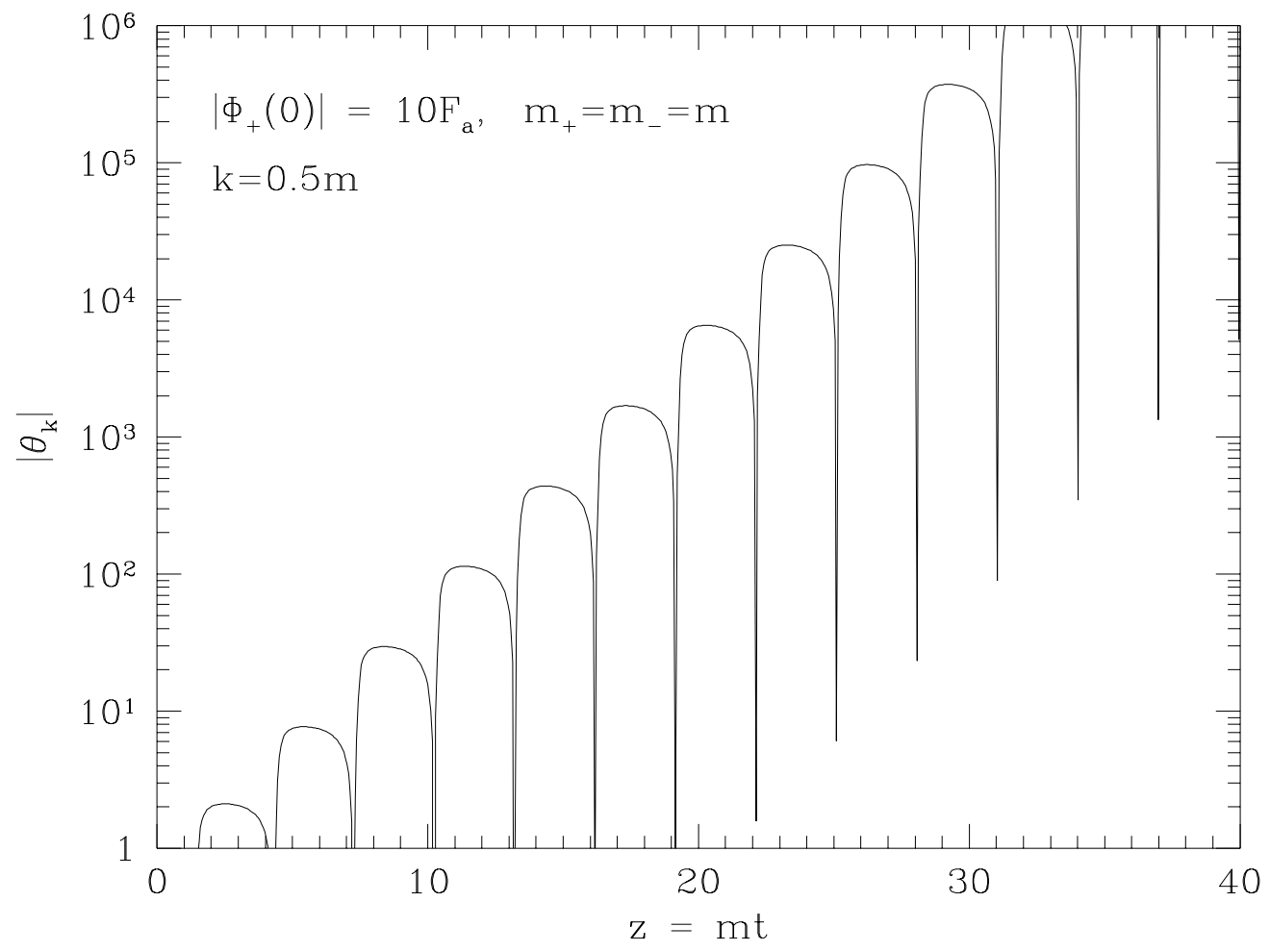

Figure 5: Time evolution of the phase fluctuations with $k=0.5 m_{+}$. We take $\left|\Phi_{+}(0)\right|=$ $10 F_{a}$ and $m=m_{+}=m_{-}$. This figure shows clearly the instability of the phase fluctuations. 